\title{
GENERIC EMBEDDINGS AND THE FAILURE OF BOX
}

\author{
DOUGLAS BURKE
}

(Communicated by Andreas R. Blass)

\begin{abstract}
We prove that if $\left\{a \subseteq \kappa^{+} \mid\right.$order type of $a$ is a cardinal $\}$ is stationary, then Jensen's principle $\square_{\kappa}$ fails. We also show that $\forall \kappa \square_{\kappa}$ is consistent with a superstrong cardinal.
\end{abstract}

\section{INTRODUCTION}

The results of this paper were motivated by the question "Where can $\omega_{1}$ be sent in Woodin's non-stationary tower?" ([W88]). A set $\mathscr{A} \subset \mathscr{P}(X)$ is stationary (in $\mathscr{P}(X))$ iff $\forall f: X^{<\omega} \rightarrow X \quad \exists a \in \mathscr{A}(a \neq X)$ such that $a$ is closed under $f$. By " $\omega_{1}$ can be sent to $\kappa$ " (in symbols $\omega_{1} \rightarrow \kappa$ ) we mean $\left\{a \subseteq \kappa \mid \operatorname{ot}(a)=\omega_{1}\right\}$ is stationary. More generally, a cardinal $\kappa$ is preserved $(\operatorname{Pr}(\kappa))$ iff $\{a \subseteq \kappa \mid \operatorname{ot}(a)$ is a cardinal $\}$ is stationary. If $\kappa$ is Ramsey, then $\omega_{1} \rightarrow \kappa$; Chang's conjecture is equivalent to $\omega_{1} \rightarrow \omega_{2}$ ([KM]). We show below that $\operatorname{Pr}\left(\kappa^{+}\right)$implies $\neg \square_{\kappa}$. (It was known that Chang's conjecture implies $\neg \square_{\omega_{1}}$.) We also show that $\forall \kappa \neg \operatorname{Pr}\left(\kappa^{+}\right)$is consistent with a superstrong cardinal (by showing that $\forall \kappa \square_{\kappa}$ is consistent with a superstrong cardinal). It is easy to see that if $\kappa$ is supercompact, then $\operatorname{Pr}\left(\kappa^{+}\right)$.

We start with some basic well-known definitions and results. A generic embedding is an elementary embedding $j: V \rightarrow(M, E)$ defined in some generic extension of $V$. We assume that the wellfounded part of $(M, E)$ is collapsed to a transitive set. If $P$ is the partial order of stationary subsets of $\mathscr{P}(\lambda)$ (ordered by inclusion) and $G \subseteq P$ is generic, then we get a generic embedding $j: V \rightarrow(M, E)$. The model $(M, E)$ is the ultrapower $V^{\mathscr{P}(\lambda)} / G$ and $\operatorname{cp}(j) \leq$ $\lambda$. Also, there is an $A \in M$ ( $A$ is [id]) such that $\{B \in M \mid M \vDash B \in A\}=j^{\prime \prime} \lambda$ (we abbreviate this by $j^{\prime \prime} \lambda \bar{\in} M$ ) (see [F] for proofs).

Lemma 1.1. Assume $j: V \rightarrow(M, E)$ is a generic embedding with $\operatorname{cp}(j) \leq \lambda$ and $j^{\prime \prime} \lambda \bar{\in} M$. Then $\lambda$ is in the wellfounded part of $M$, for all $X \subseteq \lambda$ (with $X \in V) \quad X \in M$, and $\exists \bar{j} \in M$ such that $\forall \alpha \in \lambda M \vDash " j(\alpha)=\bar{j}(\alpha)$ ".

Proof. This follows easily since $j^{\prime \prime} \lambda \bar{\epsilon} M$.

Definition 1.2. $\operatorname{Pr}(\kappa)$ means that $\{a \subset \kappa \mid \operatorname{ot}(a)$ is a cardinal $\}$ is stationary.

Lemma 1.3. The following are equivalent:

(1) $\operatorname{Pr}\left(\kappa^{+}\right)$.

Received by the editors September 18, 1993 and, in revised form, January 31, 1994.

1991 Mathematics Subject Classification. Primary 03E35, 03E55.

(c) 1995 American Mathematical Society 
(2) There is a generic embedding $j: V \rightarrow(M, E)$ such that $\operatorname{cp}(j) \leq \kappa^{+}$, $j^{\prime \prime} \kappa^{+} \bar{\epsilon} M$, and $M \vDash$ " $\kappa^{+}$is a cardinal".

Proof. (1) $\rightarrow(2)$. Force with the stationary subsets of $\mathscr{P}\left(\kappa^{+}\right)$below $\{a \subset$ $\kappa^{+} \mid \operatorname{ot}(a)$ is a cardinal $\}$. So we just need to check that $M \vDash " \kappa^{+}$is cardinal". But $M \vDash$ "ot([id]) is a cardinal", and ot([id]) is $\kappa^{+}$.

$(2) \rightarrow(1)$. Let $f:\left(\kappa^{+}\right)^{<\omega} \rightarrow \kappa^{+}$(with $f \in V$ ). In $M, j^{\prime \prime} \kappa^{+}$is closed under $j(f)$ and $\operatorname{ot}\left(j^{\prime \prime} \kappa^{+}\right)=\kappa^{+}$is a cardinal.

Clearly, $\omega_{1} \rightarrow \kappa$ implies $\operatorname{Pr}(\kappa)$. A cardinal $\kappa$ is Jónsson iff $\{a \subseteq \kappa|| a \mid=\kappa\}$ is stationary. If $C$ is a set of ordinals, then $C^{\prime}$ is all limit points of $C \cdot \square_{\kappa}$ means $\exists\left\langle C_{\alpha}: \alpha \in \kappa^{+}\right\rangle$such that:

(1) $C_{\alpha}$ is club in $\alpha$,

(2) $\beta \in\left(C_{\alpha}\right)^{\prime}$ implies $C_{\beta}=C_{\alpha} \cap \beta$,

(3) $\operatorname{cf}(\alpha)<\kappa$ implies ot $\left(C_{\alpha}\right)<\kappa$.

A cardinal $\kappa$ is superstrong if there is an elementary embedding $j: V \rightarrow M$ with $\operatorname{cp}(j)=\kappa$ and $V_{j(\kappa)} \subset M$. If $\mathbb{P}$ is a partial order, then $\mathbb{P}$ is $\alpha \quad(\alpha$ an ordinal) strategically closed iff

$$
\left(\forall p_{1} \exists p_{2} \ldots Q p_{\beta} \ldots\right)\left(p_{1} \geq p_{2} \geq \cdots \geq p_{\beta} \geq \cdots\right)
$$

where $Q$ is $\exists$ if $\beta$ is even and $\forall$ if $\beta$ is odd and the string of $\alpha$ many quantifiers is interpreted as a game. If $M, N$ are models of ZFC, then $M \sim_{\lambda} N$ means that $V_{\lambda}^{M}=V_{\lambda}^{N}$. For basic results about extenders see [MS]. For any other unexplained notions see $[\mathrm{J}]$.

\section{FAILURE OF BOX}

Theorem 2.1. Let $\kappa$ be a cardinal. If $\operatorname{Pr}\left(\kappa^{+}\right)$, then $\neg \square_{\kappa}$.

Proof. Assume that $\left\{a \subset \kappa^{+} \mid \operatorname{ot}(a)\right.$ is a card $\}$ is stationary and therefore there is a generic embedding $j: V \rightarrow(M, E)$ such that $\operatorname{cp}(j) \leq \kappa^{+}, j^{\prime \prime} \kappa^{+} \bar{\epsilon} M$, and $M \vDash$ " $\kappa^{+}$is a card". Everywhere below $\kappa^{+}$denotes the successor cardinal of $\kappa$ in $V$. We assume the wellfounded part of $M$ is collapsed to a transitive set, so by Lemma $1.1, \kappa^{+} \in M$, there is a $\bar{j} \in M$ such that $M \vDash " \bar{j}: \kappa^{+} \rightarrow$ Ord" and $\forall \alpha \in \kappa^{+} M \vDash " \bar{j}(\alpha)=j(\alpha)$ ", and for all $X \subseteq \kappa^{+}$(with $X \in V$ ) $X \in M$. Therefore $M \vDash$ " $\kappa^{+}$is a successor card" and $\operatorname{cp}(j)<\kappa^{+}$.

We may assume $j\left(\kappa^{+}\right)>\kappa^{+}$. (If $j\left(\kappa^{+}\right)=\kappa^{+}$, then $\kappa^{+}$is Jónsson and so every stationary subset of $\kappa^{+}$reflects $([\mathrm{T}])$ and therefore $\neg \square_{\kappa}$. )

Towards a contradiction assume $\left\langle C_{\alpha}: \alpha \in \kappa^{+}\right\rangle$is a $\square_{\kappa}$ sequence. Now work in $M$. So $\left\langle j(C)_{\alpha}: \alpha \in j\left(\kappa^{+}\right)\right\rangle$is a $\square_{j(\kappa)}$ sequence. Let $\gamma=\sup _{\alpha \in \kappa^{+}} \bar{j}(\alpha)$. So $j(\kappa)<\gamma<j\left(\kappa^{+}\right)$and $\operatorname{cf}(\gamma)=\kappa^{+}$. Let $\bar{\gamma}=\operatorname{ot}\left(j(C)_{\gamma}\right)$. So $\bar{\gamma} \leq j(\kappa)$ and $\operatorname{cf}(\bar{\gamma})=\kappa^{+}$.

Since $\bar{\gamma}<\gamma$ and $\operatorname{cf}(\bar{\gamma})=\kappa^{+}$, the range of $\bar{j}$ is bounded in $\bar{\gamma}$, say $\alpha_{b}$ is the bound. Choose $\nu \in\left(j(C)_{\gamma}\right)^{\prime} \cap\left(\bar{j}^{\prime \prime} \kappa^{+}\right)^{\prime}$ with ot $\left(j(C)_{\gamma} \cap \nu\right)>\alpha_{b}$. Let $\eta$ be minimal such that $\bar{j}(\eta) \geq \nu\left(\right.$ so $\left.\sup \bar{j}^{\prime \prime} \eta=\nu\right)$. Since $j(C)_{\nu}=j(C)_{\gamma} \cap \nu$, we have that $\alpha_{b}<\operatorname{ot}\left(j(C)_{\nu}\right)<\bar{\gamma}$. If $\bar{j}(\eta)=\nu$, then $j(C)_{\nu}=j\left(C_{\eta}\right)$ and so $\operatorname{ot}\left(j(C)_{\nu}\right) \in$ range of $j$, contradiction. So $\bar{j}(\eta)>\nu$. Since $\sup \bar{j}^{\prime \prime} \eta=\nu$, we have $j\left(C_{\eta}\right) \cap \nu=j(C)_{\nu}$. Choose $\rho$ such that $\rho<\eta$ and $\operatorname{ot}\left(j(C)_{\nu} \cap \bar{j}(\rho)\right)>\alpha_{b}$. Since $j\left(C_{\eta}\right) \cap \nu=j(C)_{\nu}$ and $\bar{j}(\rho)<\nu$, ot $\left(j\left(C_{\eta}\right) \cap \bar{j}(\rho)\right)=\operatorname{ot}\left(j(C)_{\nu} \cap \bar{j}(\rho)\right)$. But ot $\left(j\left(C_{\eta}\right) \cap \bar{j}(\rho)\right)=j\left(\operatorname{ot}\left(C_{\eta} \cap \rho\right)\right)$, a contradiction. 
In [LMS] they show it is consistent (from a 2-huge) that $\omega_{1} \rightarrow \omega_{\omega+1}$. The above theorem and work of Steel, Mitchell and Schimmerling ([MS], [S], [MSS]) show that $\omega_{1} \rightarrow \omega_{\omega+1}$ (or just $\operatorname{Pr}\left(\omega_{\omega+1}\right)$ ) gives an inner model with a cardinal $\kappa$ such that $o(\kappa)=\kappa^{++}$. (It is shown in [S] that $\neg \square_{\aleph_{\omega}}$ gives such a model.) Schimmerling also gets an inner model of a Woodin cardinal from the failure of weaker principles; this suggests the following questions. Does $\omega_{1} \rightarrow \omega_{\omega+1}$ imply the failure of the weak square property $\left(\neg \square_{\aleph_{\omega}}^{*}\right)$ ? Does $\omega_{1} \rightarrow \omega_{\omega+1}$ imply stationary reflection at $\omega_{\omega+1}$ ?

\section{BOX AND SUPERSTRONG CARDINALS}

Theorem 3.1. There is a class forcing $\mathbb{P}$ such that $V^{\mathbb{P}} \vDash$ "ZFC plus $\forall \operatorname{card} \kappa$ $\square_{\kappa}$ ". If $V \vDash$ " $\delta$ is superstrong", then $V^{\mathbb{P}} \vDash$ " $\delta$ is superstrong".

Proof. $\mathbb{P}$ will be the Easton support iteration of the standard forcings for adding a square sequence. The main point is to check that superstrong cardinals are preserved. For any cardinal $\kappa$ let $\mathbb{B}_{\kappa}=$ the set of all functions $p$ such that:

(1) $\operatorname{dom}(p)=$ the limit ordinals $\leq \gamma$ for some $\gamma \in \kappa^{+}$,

(2) $p(\alpha) \subset \alpha$ is club,

(3) $\operatorname{cf}(\alpha)<\kappa \Rightarrow \operatorname{ot}(p(\alpha))<\kappa$,

(4) if $\beta \in p(\alpha)^{\prime}$, then $p(\beta)=p(\alpha) \cap \beta$.

It is easy to check that $\mathbb{B}_{\kappa}$ is $\kappa+1$ strategically closed (and so adds no new $\kappa$ sequences of ordinals) and that $V^{\mathbb{B}_{\kappa}} \vDash \square_{\kappa}$ (see [J], p. 255).

Given any ordinal $\beta$ define an iteration $\mathbb{P}(\beta)$ with Easton support (direct limits at inaccessible cardinals, inverse limits everywhere else) by letting $\mathbb{Q}_{\alpha}$ name $\{1\}$ if $\alpha<\beta$ or if $V^{\mathbb{P}(\beta)\lceil\alpha} \vDash " \alpha$ is not a cardinal". Otherwise, $\mathbb{Q}_{\alpha}$ names $\mathbb{B}_{\alpha}$ (in $\left.V^{\mathbb{P}(\beta) \mid \alpha}\right)$. The forcing $\mathbb{P}$ we use is $\mathbb{P}\left(\omega_{1}\right)$. The basic factor lemma (see [B], 5.1-5.4) gives that for any $\gamma, \mathbb{P}(\beta) \cong \mathbb{P}(\beta) \mid \gamma^{*} \mathbb{P}(\gamma)$ (where $\mathbb{P}(\gamma)$ names $\mathbb{P}(\gamma)$ in $\left.V^{\mathbb{P}(\beta) \mid \gamma}\right)$. Also, for any Mahlo cardinal $\gamma, \mathbb{P}(\beta) \mid \gamma$ has the $\gamma$-cc $([\mathrm{B}], 2.4)$.

Claim. For any $\gamma, \mathbb{P}(\gamma)$ adds no new $\gamma$ sequences.

Proof of Claim. We will show that $\forall \alpha \mathbb{P}(\gamma) \mid \alpha$ is $\gamma+1$ strategically closed, and so the claim follows. We inductively define winning strategies $\tau_{\alpha}$ for $\mathbb{P}(\gamma)\lceil\alpha$ such that:

(1) If $p_{0}, p_{1}, \ldots$ is any play according to $\tau_{\alpha}$ and $\beta<\alpha$, then $p_{0} \mid \beta$, $p_{1} \uparrow \beta, \ldots$ is according to $\tau_{\beta}$.

(2) If $p_{0}, p_{1}, \ldots$ is any partial play according to $\tau_{\alpha}$ and for some $\beta<\alpha$ and for all $i \quad p_{i}=p_{i} \uparrow \beta \sim\langle 1, \ldots$,$\rangle , then \tau_{\alpha}\left(p_{0}, \ldots\right)=\tau_{\alpha}\left(p_{o}, \ldots\right) \uparrow$ $\beta \sim\langle 1, \ldots\rangle$.

The construction at limit stages is easy (note that if we do not have an inverse limit at $\alpha$, then $\operatorname{cf}(\alpha)=\alpha>\gamma$ ). For successor stages assume we have $\tau_{\alpha}$ and let $\dot{\sigma}_{\alpha}$ be a name such that

$$
\Vdash_{\mathbb{P}(\gamma)\lceil\alpha} \text { “ } \dot{\sigma}_{\alpha} \text { witnesses } \dot{\mathbb{Q}}_{\alpha} \text { is } \gamma+1 \text { strategically closed". }
$$

(We may assume if $I$ plays only 1 's, then $I I$ 's response with $\dot{\sigma}_{\alpha}$ is 1 .) Now let $\tau_{\alpha+1}\left(\left(p_{0}, q_{0}\right), \ldots\right)=\left(\tau_{\alpha}\left(p_{0}, \ldots\right), \dot{\sigma}_{\alpha}\left(q_{0}, \ldots\right)\right)$. This completes the proof of the claim.

It is easy to see that $V^{\mathbb{P}} \vDash Z F C$ (see [J], p. 196). Also $V^{\mathbb{P}} \vDash " \forall$ card $\kappa \square_{\kappa}$ ": Let $G$ be generic for $\mathbb{P}$. Assume $V[G] \vDash$ " $\kappa$ is a card ". So $V[G\lceil\kappa] \vDash$ " $\kappa$ is 
a card". Hence $\mathbb{Q}_{\kappa}$ names $\mathbb{B}_{\kappa}$ in $V\left[G\lceil\kappa]\right.$. Let $\kappa^{+}$be the successor of $\kappa$ in $V[G \backslash \kappa]$. So

$$
V\left[G\lceil\kappa+1] \vDash \text { " } \kappa \text { and } \kappa^{+} \text {are still cardinals and } \square_{\kappa}\right. \text { ". }
$$

Since in $V\left[G\lceil\kappa+1], \mathbb{P}(\kappa+1) \cong \mathbb{P}\left(\kappa^{+}\right), \mathbb{P}(\kappa+1)\right.$ adds no new $\kappa^{+}$sequences to $V[G \backslash \kappa+1]$. Hence $v[G] \vDash " \kappa \& \kappa^{+}$are cardinals and $\square_{\kappa}$ ".

Finally, suppose $V \vDash \kappa$ is superstrong. Let $j: V \rightarrow M$ witness this (so $\left.V_{j(\kappa)} \subset M\right)$. Let $G \subset \mathbb{P}$ be generic. So $V[G]=V\left[G_{1}\right]\left[H_{1}\right]\left[H_{2}\right]$ where $G_{1} \subset \mathbb{P}$ । $\kappa, H_{1} \subset \mathbb{P}(\kappa) \uparrow j(\kappa)$ and $H_{2} \subset \mathbb{P}(j(\kappa))$ come from $G$. Let $\widetilde{H}_{1}=\left\{p \in H_{1} \mid p\right.$ ends in a tail of 1 's $\}$.

Claim. $G_{1} * \widetilde{H}_{1}$ is generic for $j(\mathbb{P} \mid \kappa)$ over $M$.

Proof of Claim. Suppose $D \subset j(\mathbb{P} \mid \kappa)$ is dense and in $M$. Since $M \vDash$ " $j(\kappa)$ is Mahlo" (it is superstrong), there is an inaccessible $\lambda<j(\kappa)$ such that $\{p \in \mathbb{P} \mid$ $\lambda \mid p-\langle 1, \ldots\rangle \in D\}$ is dense in $\mathbb{P} \mid \lambda$. (Note that $(\mathbb{P} \mid \lambda)^{M}=\mathbb{P} \mid \lambda$.) So there is a $p \in G\left\lceil\lambda\right.$ such that $p-\langle 1, \ldots\rangle \in D$. Hence $p-\langle 1, \ldots\rangle \in D \cap\left(G_{1} * \widetilde{H}_{1}\right)$. This completes the proof of the claim.

Hence we can extend $j$ to an elementary embedding $\tilde{j}: V\left[G_{1}\right] \rightarrow M\left[G_{1}\right]\left[\tilde{H}_{1}\right]$. Now let $E$ be the $(\kappa, j(\kappa))$ extender derived from $\tilde{j}$. So we get the following commutative diagram:

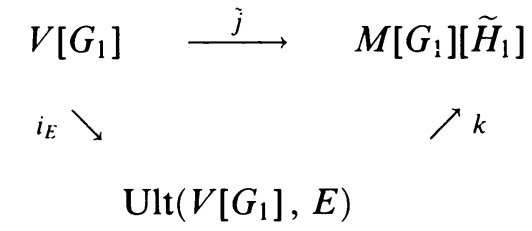

Note that $i_{E}(\kappa)=j(\kappa), \operatorname{cp}(k)>j(\kappa)$ and $M\left[G_{1}\right]\left[\tilde{H}_{1}\right] \sim_{j(\kappa)} \operatorname{Ult}\left(V\left[G_{1}\right], E\right)$. Since $V[G]$ adds no new $\kappa$ sequences (of ordinals) to $V\left[G_{1}\right], \operatorname{Ult}(V[G], E$ ) makes sense and is well founded. Note that $V\left[G_{1}\right] \vDash \kappa$ is strongly inaccessible and so $V[G] \sim_{\kappa+1} V\left[G_{1}\right]$ and therefore $\operatorname{Ult}(V[G], E) \sim_{j(\kappa)} \operatorname{Ult}\left(V\left[G_{1}\right], E\right)$ (and $i_{E}^{V[G]}(\kappa)=j(\kappa)$ also). We now show that $V[G] \sim_{j(\kappa)} M\left[G_{1}\right]\left[\widetilde{H}_{1}\right]$ and so $V[G] \vDash \kappa$ is superstrong. Clearly $M\left[G_{1}\right]\left[\widetilde{H}_{1}\right]_{j(\kappa)} \subset V[G]$. So suppose $x \in$ $(V[G])_{j(\kappa)}$. We may assume that $x \subset \lambda$ where $\lambda<j(\kappa)$ is a cardinal $(j(\kappa)$ is a strong limit cardinal in $V[G]$ since it is superstrong in $M$ and therefore a limit of Mahlos in $V)$. So $x \in V\left[G\lceil\lambda]\right.$. Hence $x \in V_{j(\kappa)}\left[G\lceil\lambda] \subset M\left[G_{1}\right]\left[\tilde{H}_{1}\right]\right.$.

This theorem shows that we cannot prove there is a successor cardinal $\kappa^{+}$ such that $\omega_{1} \rightarrow \kappa^{+}$from a superstrong cardinal. Does a supercompact cardinal imply the existence of such a $\kappa^{+}$?

\section{ACKNOWLEDGMENT}

The author thanks Ernest Schimmerling and James Cummings for helpful discussions.

\section{REFERENCES}

[B] J. Baumgartner, Iterated forcing (A. R. D. Mathis, ed.), Surveys in Set Theory, Cambridge Univ. Press, Cambridge, 1983.

[F] M. Foreman, Potent axioms, Trans. Amer. Math. Soc. 294 (1986), 1-28. 
[J] T. J. Jech, Set theory, Academic Press, New York, 1978.

[KM] A. Kanamori and M. Magidor, The evolution of large cardinal axioms in set theory, Lecture Notes in Math., vol. 669, Springer-Verlag, Berlin and New York, 1978, pp. 99-275.

[LMS] J.-P. Levinski, M. Magidor, and S. Shelah, Changs conjecture for $\aleph_{\omega+1}$, Israel J. Math. 69 (1990), 161-172.

[MS] D. A. Martin and J. R. Steel, A proof of projective determinancy, J. Amer. Math. Soc. 2 (1989), 71-125.

[MSS] W. J. Mitchell, E. Schimmerling, and J. R. Steel, The covering lemma up to one Woodin cardinal (to appear).

[MiS] W. J. Mitchell and J. R. Steel, Fine structure and iteration trees, Lecture Notes in Logic, vol. 3, Springer-Verlag, Berlin, 1994.

[S] E. Schimmerling, Combinatorial principles in the core model for one Woodin cardinal, Ph.D. thesis, UCLA, 1992.

[T] J. Tryba, No Jónsson filters over $\aleph_{\omega}$, J. Symbolic Logic 52 (1987), 51-53.

[W88] H. Woodin, Supercompact cardinals, sets of reals and weakly homogeneous trees, Proc. Nat. Acad. Sci. U.S.A. 85 (1988), 6587-6591.

Department of Mathematics, University of North Texas, Denton, TeXas 76203

Current address: Department of Mathematical Sciences, University of Nevada, Las Vegas, Nevada 89154

E-mail address: dburke@nevada.edu 